\title{
Exact Shape Functions for Timoshenko Beam Element
}

\author{
Sri Tudjono ${ }^{1}$, Aylie Han ${ }^{1}$, Dinh-Kien Nguyen ${ }^{2}$, Shota Kiryu ${ }^{3}$, Buntara S. Gan ${ }^{3 *}$ \\ ${ }^{I}$ Department of Civil Engineering, Diponegoro University, Indonesia \\ ${ }^{2}$ Institute of Mechanics, Vietnam Academy of Science and Technology, Vietnam \\ ${ }^{3}$ Department of Architecture, College of Engineering, Nihon University, Japan
}

\begin{abstract}
This paper derives exact shape functions for both non-uniform (non-prismatic section) and inhomogeneous (functionally graded material) Timoshenko beam element formulation explicitly. In this paper, the shape functions formula embedded the explicit functions and its derivatives describing the non-uniformity and inhomogeneity of a beam element. The shape functions are made interdependent by requiring them to satisfy three homogeneous differential equations associated with the Timoshenko's beam theory. With the formulated axial, transverse and rotational displacement shape functions, the stiffness and mass matrices and consistent force vector for a two-node Timoshenko beam element are developed based on Hamilton's principle. Comparison studies with reference work on the accuracy and computational efficiency for non-uniform and inhomogeneous Timoshenko beam structures are highlighted. Static and vibrational analyses of the beams element by using the exact shape functions can predict the displacement, and natural frequencies of nonuniform and inhomogeneous Timoshenko beams by using only one/the least element accurately.
\end{abstract}

Keywords : Exact shape functions, Timoshenko beam, FEM, Functionally graded material

\section{Introduction}

In the state-of-the-art of computational beam element, the polynomial function assumptions are most usually used. However, these functions were developed base on the uniform cross-section and homogenous material. By using these shape functions, the analyses of beam elements require a significant number of element divisions to study the behavior of beam with non-uniformity and inhomogeneity problems accurately [1-12]. The latest accomplishment for developing the exact solutions of the shape functions makes use of the power series method [13-14], basic displacement function [15] and Taylor series expansion [16-17] approximations are reported.

Given the fact that the finite element method has been a widely used technique for structural analysis, it is necessary to develop reliable and accurate displacement functions [18-26] for the beam element. With the proper shape functions in the finite element formulations, the mass, stiffness matrices and loading vectors can be constructed consistently. In this paper, the formulation for exact shape functions for both non-uniform and inhomogeneous Timoshenko beam element are derived based on Hamilton's principle.

\section{Formulation of Exact Displacement Functions}

The Euler-Lagrange equations [27] for the Timoshenko beams can be obtained from

$$
\begin{aligned}
& \frac{\partial}{\partial x}\left(E(x) A(x) \frac{\partial u}{\partial x}\right)=0 \\
& \frac{\partial}{\partial x}\left(\kappa G(x) A(x)\left(\frac{\partial w}{\partial x}-\theta\right)\right)=0 \\
& \frac{\partial}{\partial x}\left(E(x) I(x) \frac{\partial \theta}{\partial x}\right)+\kappa G(x) A(x)\left(\frac{\partial w}{\partial x}-\theta\right)=0
\end{aligned}
$$

where, $u, w$ and $\theta$ are the axial displacement, transversal displacement and rotation. $E(x), A(x), G(x)$ and $\kappa$ are the elastic modulus, section area and shear modulus as the function of $x$ and shear correction factor, respectively.

By integration, (1a) becomes,

$$
E(x) A(x) \frac{\partial u}{\partial x}=k_{1}
$$

where $k_{l}$ is the indefinite integration integral constant. Next, solving for $u(x)$, 


$$
u(x)=k_{1} \int \frac{1}{E(x) A(x)} d x+k_{2}=k_{1} \psi_{1}+k_{2}
$$

defining,

$$
\psi_{1}=\int \frac{1}{E(x) A(x)} d x
$$

Imposing the boundary conditions of horizontal displacements at both end nodes of the beam results in, $u(x=0)=u_{1}, u(x=L)=u_{2}$.

Solving for $k_{1}, k_{2}$ from (2) and (3), then substituting back the results into (2), the exact displacement functions and its first order derivative of the axial displacement of the beam can be obtained as

$$
\begin{aligned}
& u(x)=\left[\begin{array}{ll}
N_{u 1} & N_{u 2}
\end{array}\right]\left\{\begin{array}{l}
u_{1} \\
u_{2}
\end{array}\right\} \\
& N_{u 1}=\frac{\psi_{1}-\psi_{1 L}}{\psi_{10}-\psi_{1 L}} \\
& N_{u 2}=\frac{-\psi_{1}+\psi_{10}}{\psi_{10}-\psi_{1 L}} \\
& \frac{d N_{u 1}}{d x}=\frac{\psi_{1}^{\prime}}{\psi_{10}-\psi_{1 L}} \\
& \frac{d N_{u 2}}{d x}=\frac{-\psi_{1}^{\prime}}{\psi_{10}-\psi_{1 L}}
\end{aligned}
$$

where $\psi_{10}=\left.\psi_{1}\right|_{x=0}, \psi_{1 L}=\left.\psi_{1}\right|_{x=L}$.

The transverse displacement is assumed in polynomial equation as follows

$$
\begin{aligned}
& w(x)=a_{0}+a_{1} x+\frac{a_{2}}{2} x^{2}+\frac{a_{3}}{6} x^{3} \\
& \frac{\partial w}{\partial x}=a_{1}+a_{2} x+\frac{a_{3}}{2} x^{2} \\
& \frac{\partial^{2} w}{\partial x^{2}}=a_{2}+a_{3} x
\end{aligned}
$$

The solution of transverse homogeneous equilibrium equation of (1b) can be obtained from

$$
\begin{aligned}
& \kappa G(x) A(x)\left(\frac{\partial w}{\partial x}-\theta\right)=c_{1} \\
& w(x)=c_{1} \psi_{4}+\int \theta(x) d x+c_{4} \\
& \theta(x)=-c_{1} \frac{1}{\kappa G(x) A(x)}+\frac{\partial w}{\partial x}
\end{aligned}
$$

Replacing (6) into (7), results in

$$
\begin{aligned}
& \theta(x)=-c_{1} \psi_{4}^{\prime}+a_{1}+a_{2} x+\frac{a_{3}}{2} x^{2} \\
& \frac{\partial \theta}{\partial x}=-c_{1} \psi_{4}^{\prime \prime}+a_{2}+a_{3} x \\
& \frac{\partial^{2} \theta}{\partial x^{2}}=-c_{1} \psi_{4}^{\prime \prime \prime}+a_{3}
\end{aligned}
$$


Replacing (8) into (7), results in

$$
\begin{aligned}
w(x) & =c_{1} \psi_{4}+\int \theta(x) d x+c_{4} \\
& =c_{1} \psi_{4}+\int\left(-c_{1} \psi_{4}^{\prime}+a_{1}+a_{2} x+\frac{a_{3}}{2} x^{2}\right) d x+c_{4} \\
& =a_{1} x+\frac{a_{2}}{2} x^{2}+\frac{a_{3}}{6} x^{3}+c_{4}
\end{aligned}
$$

Hence, (1c) can be written as

$$
\begin{aligned}
& \frac{\partial}{\partial x}\left(E(x) I(x) \frac{\partial \theta}{\partial x}\right)+c_{1}=0 \\
& \frac{\partial \theta}{\partial x}=-c_{1} \frac{x}{E(x) I(x)}+c_{2} \frac{1}{E(x) I(x)}=-c_{1} \psi_{3}^{\prime}+c_{2} \psi_{2}^{\prime} \\
& \theta(x)=-c_{1} \psi_{3}+c_{2} \psi_{2}+c_{3}
\end{aligned}
$$

the second derivative can be given by,

$$
\frac{\partial^{2} \theta}{\partial x^{2}}=-c_{1} \psi_{3}^{\prime \prime}+c_{2} \psi_{2}^{\prime}
$$

where $c_{i}(i=1,2,3,4)$ and $a_{i}(i=1,2,3,4)$ are the polynomial constants. Defining,

$$
\begin{aligned}
& \psi_{2}=\int \frac{1}{E(x) I(x)} d x, \\
& \psi_{3}=\int \frac{x}{E(x) I(x)} d x, \\
& \psi_{4}=\int \frac{1}{\kappa G(x) A(x)} d x
\end{aligned}
$$

Collecting rotation and its derivatives from (8) and (10), as well as transverse displacement between (6) and (9), we have relationship between $a_{i}$ and $c_{i}$

$$
\{\mathbf{c}\}=\left[\mathbf{M}_{\mathrm{ac}}\right]^{-1}\{\mathbf{a}\}
$$

Imposing the boundary conditions of vertical displacements and rotations at both end nodes of the beam results in, $w(x=0)=w_{1}, w(x=L)=w_{2}$ and $\theta(x=0)=\theta_{1}, \theta(x=L)=\theta_{2}$.

$$
\left\{\begin{array}{l}
c_{1} \\
c_{2} \\
c_{3} \\
c_{4}
\end{array}\right\}=\left[\mathbf{M}_{\mathrm{w \theta c}}\right]^{-1}\left\{\begin{array}{l}
w_{1} \\
\theta_{1} \\
w_{2} \\
\theta_{2}
\end{array}\right\}
$$

By substituting $c_{i}$, the expressions for transverse and rotational shape functions are as follow,

$$
\begin{aligned}
& w(x)=\left[\begin{array}{llll}
N_{w 1} & N_{w 2} & N_{w 3} & N_{w 4}
\end{array}\right]\left\{\begin{array}{l}
w_{1} \\
\theta_{1} \\
w_{2} \\
\theta_{2}
\end{array}\right\} \\
& \theta(x)=\left[\begin{array}{llll}
N_{\theta 1} & N_{\theta 2} & N_{\theta 3} & N_{\theta 4}
\end{array}\right]\left\{\begin{array}{l}
w_{1} \\
\theta_{1} \\
w_{2} \\
\theta_{2}
\end{array}\right\}
\end{aligned}
$$

where the rotational shape functions and their derivatives are given by 


$$
\begin{aligned}
& N_{\theta 1}=\frac{1}{\Phi L}\left[6\left(\psi_{2 L}\left(-\psi_{3}+\psi_{30}\right)+\psi_{20}\left(\psi_{3}-\psi_{3 L}\right)+\psi_{2}\left(-\psi_{30}+\psi_{3 L}\right)\right)\right] \\
& N_{\theta 2}=-\frac{1}{\Phi}\left[\begin{array}{l}
\left(\psi_{3}-\psi_{3 L}\right) L\left(3 \psi_{2 L}^{\prime}-\psi_{2 L}^{\prime \prime} L\right)+\psi_{2}\left(-6 \psi_{4 L}^{\prime}+L\left(-3 \psi_{3 L}^{\prime}+3 \psi_{4 L}^{\prime \prime}+\right.\right. \\
\left.\left.\psi_{3 L}^{\prime \prime} L-\psi_{4 L}^{\prime \prime} L\right)\right)+\psi_{2 L}\left(6 \psi_{4 L}^{\prime}+L\left(3 \psi_{3 L}^{\prime}-3 \psi_{4 L}^{\prime \prime}-\psi_{3 L}^{\prime \prime} L+\psi_{4 L}^{\prime \prime \prime} L\right)\right)
\end{array}\right] \\
& N_{\theta 3}=-N_{\theta 1}\left[\begin{array}{l}
6 \psi_{2 L}\left(\psi_{3}-\psi_{30}\right)+6 \psi_{2} \psi_{30}-6 \psi_{2} \psi_{3 L}+6 \psi_{2} \psi_{4 L}^{\prime}-3 \psi_{2 L}^{\prime} \psi_{3 L} \\
+3 \psi_{2 L}^{\prime} \psi_{30} L+3 \psi_{2} \psi_{3 L}^{\prime} L-3 \psi_{2} \psi_{4 L}^{\prime \prime} L+\psi_{2 L}^{\prime \prime} \psi_{3} L^{2}-\psi_{2 L}^{\prime \prime} \psi_{30} L^{2} \\
-\psi_{2} \psi_{3 L}^{\prime \prime} L^{2}+\psi_{2} \psi_{4 L}^{\prime \prime \prime} L^{2}+\psi_{20}\left(-6 \psi_{3}+6 \psi_{3 L}-6 \psi_{4 L}^{\prime}-3 \psi_{3 L}^{\prime} L\right. \\
\left.+3 \psi_{4 L}^{\prime \prime} L+\psi_{3 L}^{\prime \prime} L^{2}-\psi_{4 L}^{\prime \prime \prime} L^{2}\right)
\end{array}\right] \\
& N_{\theta 4}=-\frac{1}{\Phi L}\left[\begin{array}{l}
x\left(\psi_{20}-\psi_{2 L}\right)\left(\begin{array}{l}
3 \psi_{4}^{\prime \prime} x+6 \psi_{3}-6 \psi_{4}^{\prime} \\
-3 \psi_{3}^{\prime} x+\psi_{3}^{\prime \prime} x^{2}-\psi_{4}^{\prime \prime \prime} x^{2}
\end{array}\right)-6 x\left(\psi_{20} \psi_{3 L}+\psi_{2 L} \psi_{30}\right)-\Phi \\
-\left(\psi_{30}-\psi_{3 L}\right)\left(6 \psi_{2} x-3 \psi_{2}^{\prime} x^{2}+\psi_{2}^{\prime \prime} x^{3}\right)
\end{array}\right.
\end{aligned}
$$

$$
\begin{aligned}
& N_{w 2}=-\frac{1}{6 \Phi}\left[\begin{array}{l}
\left(3 \psi_{2}^{\prime} x-\psi_{2}^{\prime \prime} x^{2}\right)\left(\begin{array}{l}
-6 \psi_{4 L}^{\prime}-3 \psi_{3 L}^{\prime} L \\
+3 \psi_{4 L}^{\prime \prime} L+\psi_{3 L}^{\prime \prime} L^{2} \\
-\psi_{4 L}^{\prime \prime \prime} L^{2}
\end{array}\right)+6 x\left(\begin{array}{l}
-3 \psi_{2 L}^{\prime} \psi_{3 L}+3 \psi_{2 L}^{\prime} \psi_{3 L} L \\
+3 \psi_{2}^{\prime} \psi_{4 L}^{\prime}+\psi_{2 L}^{\prime \prime} \psi_{3} L^{2} \\
-\psi_{2 L}^{\prime \prime} \psi_{3 L} L^{2}-\psi_{2 L}^{\prime \prime} \psi_{4}^{\prime} L^{2} \\
+\psi_{2}\left(6 \psi_{4 L}^{\prime}+L\left(3 \psi_{3 L}^{\prime}\right.\right. \\
\left.-3 \psi_{4 L}^{\prime \prime}-\psi_{x}^{\prime \prime} L+\psi_{4 L}^{\prime \prime \prime} L\right)
\end{array}\right) \\
-6 \psi_{2 L}\left(\begin{array}{l}
6 \psi_{4 L}^{\prime}+L\left(3 \psi_{3 L}^{\prime}-3 \psi_{4 L}^{\prime \prime}\right. \\
\left.-\psi_{3 L}^{\prime \prime} L+\psi_{4 L}^{\prime \prime \prime} L\right)
\end{array}\right)+x\left(3 \psi_{2 L}^{\prime}-\psi_{2 L}^{\prime \prime}\right)\left(\begin{array}{l}
3 \psi_{3 L}^{\prime}-3 \psi_{4 L}^{\prime \prime} \\
-\psi_{3 L}^{\prime \prime} x+\psi_{4 L}^{\prime \prime \prime} x
\end{array}\right)
\end{array}\right] \\
& N_{w 3}=\frac{1}{\Phi L}\left[\begin{array}{l}
x\left(\left(\psi_{30}-\psi_{3 L}\right)\left(6 \psi_{2}-3 \psi_{2}^{\prime} x+\psi_{2}^{\prime \prime} x^{2}\right)+\left(\psi_{2 L}-\psi_{20}\right)\left(6 \psi_{3}-6 \psi_{4}^{\prime}-3 \psi_{3}^{\prime} x\right.\right. \\
\left.+3 \psi_{4}^{\prime \prime} x+\psi_{3}^{\prime \prime} x^{2}-\psi_{4}^{\prime \prime \prime} x^{2}\right)+6\left(\psi_{20} \psi_{3 L}-\psi_{2 L} \psi_{30}\right)
\end{array}\right] \\
& N_{w 4}=-\frac{x}{6 \Phi}\left[\begin{array}{l}
\left(6 \psi_{2}+\psi_{2}^{\prime \prime} x^{2}\right)\left(\begin{array}{l}
6 \psi_{30}-6 \psi_{3 L}+6 \psi_{4 L}^{\prime} \\
+3 \psi_{3 L}^{\prime} L-3 \psi_{4 L}^{\prime \prime} L \\
-\psi_{3 L}^{\prime \prime} L^{2}+\psi_{4 L}^{\prime \prime} L^{2}
\end{array}\right)+\left(3 \psi_{2 L}^{\prime}-\psi_{2 L}^{\prime \prime}\right)\left(\begin{array}{l}
-6 \psi_{3 L}+6 \psi_{30} L \\
+6 \psi_{4 L}^{\prime}+3 \psi_{3 L}^{\prime} x \\
-3 \psi_{4 L}^{\prime \prime} x-\psi_{3 L}^{\prime \prime} x^{2}+\psi_{4 L}^{\prime \prime \prime} x^{2}
\end{array}\right) \\
+\left(3 \psi_{2}^{\prime} x+6 \psi_{20}\right)\left(\begin{array}{l}
-6 \psi_{4 L}^{\prime}-3 \psi_{3 L}^{\prime} L \\
+3 \psi_{4 L}^{\prime \prime} L \\
+\psi_{3 L}^{\prime \prime} L^{2}-\psi_{4 L}^{\prime \prime \prime} L^{2}
\end{array}\right)+6\left(\psi_{2 L}-\psi_{20}\right)\left(\begin{array}{l}
6 \psi_{3}-6 \psi_{3 L}-6 \psi_{4}^{\prime} \\
-3 \psi_{3}^{\prime} x+3 \psi_{4}^{\prime \prime} x \\
+\psi_{3}^{\prime \prime} x^{2}-\psi_{4}^{\prime \prime \prime} x^{2}
\end{array}\right)
\end{array}\right]
\end{aligned}
$$

with their derivatives,

$$
\begin{aligned}
& \frac{\partial N_{\theta 1}}{\partial x}=\frac{1}{\Phi L}\left[6\left(\left(\psi_{20}-\psi_{2 L}\right) \psi_{3}^{\prime}+\psi_{2}^{\prime}\left(-\psi_{30}+\psi_{3 L}\right)\right)\right] \\
& \frac{\partial N_{\theta 2}}{\partial x}=\frac{1}{\Phi}\left[\psi_{2}^{\prime}\left(\begin{array}{l}
6 \psi_{4 L}^{\prime}+L\left(3 \psi_{3 L}^{\prime}\right. \\
\left.-3 \psi_{4 L}^{\prime \prime}-\psi_{3 L}^{\prime \prime} L+\psi_{4 L}^{\prime \prime \prime} L\right)
\end{array}\right)+\psi_{3 L}^{\prime}\left(-3 \psi_{2 L}^{\prime}+\psi_{2 L}^{\prime \prime} L\right)\right] \\
& \frac{\partial N_{\theta 3}}{\partial x}=-\frac{\partial N_{\theta 1}}{\partial x} \\
& \frac{\partial N_{\theta 3}}{\partial x}=-\frac{1}{\Phi}\left[\psi_{3}^{\prime}\left(\begin{array}{l}
-6 \psi_{20}+6 \psi_{2 L} \\
-3 \psi_{2 L}^{\prime} L+\psi_{2 L}^{\prime \prime} L^{2}
\end{array}\right)+\psi_{2}^{\prime}\left(\begin{array}{l}
6 \psi_{30}-6 \psi_{3 L}+6 \psi_{4 L}^{\prime}+3 \psi_{3 L}^{\prime} L \\
-3 \psi_{4 L}^{\prime \prime} L-\psi_{3 L}^{\prime \prime} L^{2}+\psi_{4 L}^{\prime \prime \prime} L^{2}
\end{array}\right)\right]
\end{aligned}
$$




$$
\begin{aligned}
& \frac{\partial N_{w 1}}{\partial x}=\frac{1}{\Phi L}\left[6\left(\psi_{2}\left(-\psi_{30}+\psi_{3 L}\right)+\psi_{20}\left(\psi_{3}-\psi_{3 L}-\psi_{4}^{\prime}\right)+\psi_{2 L}\left(-\psi_{3}+\psi_{30}+\psi_{4}^{\prime}\right)\right)\right] \\
& \frac{\partial N_{w 2}}{\partial x}=-\frac{1}{\Phi}\left[\begin{array}{l}
\left(\psi_{3}-\psi_{3 L}-\psi_{4}^{\prime}\right) L\left(3 \psi_{2 L}^{\prime}-\psi_{2 L}^{\prime \prime} L\right)+\psi_{2}\left(\begin{array}{l}
-6 \psi_{4 L}^{\prime}+L\left(-3 \psi_{3 L}^{\prime}\right. \\
\left.+3 \psi_{4 L}^{\prime \prime}+\psi_{3 L}^{\prime \prime} L-\psi_{4 L}^{\prime \prime \prime} L\right)
\end{array}\right) \\
+\psi_{2} L\left(6 \psi_{4 L}^{\prime}+L\left(3 \psi_{3 L}^{\prime}-3 \psi_{4 L}^{\prime \prime}-\psi_{3 L}^{\prime \prime} L+\psi_{4 L}^{\prime \prime \prime} L\right)\right)
\end{array}\right] \\
& \frac{\partial N_{w 3}}{\partial x}=-\frac{\partial N_{w 1}}{\partial x} \\
& \frac{\partial N_{w 4}}{\partial x}=-\frac{1}{\Phi}\left[\begin{array}{l}
\left(6 \psi_{2 L}-6 \psi_{20}+\psi_{2 L}^{\prime \prime}\right)\left(\psi_{3}-\psi_{30}-\psi_{4}^{\prime}\right) \\
+\left(\psi_{2}-\psi_{20}\right)\left(6 \psi_{4 L}^{\prime}+3 \psi_{3 L}^{\prime} L-3 \psi_{4 L}^{\prime \prime} L-3 \psi_{3 L}^{\prime \prime} L^{2}+4 \psi_{4 L}^{\prime \prime \prime} L^{2}\right) \\
+6 \psi_{2}\left(\psi_{30}-\psi_{3 L}\right)+3 \psi_{2 L}^{\prime}\left(-\psi_{3 L}+\psi_{30} L+\psi_{4 L}^{\prime}\right)
\end{array}\right]
\end{aligned}
$$

where,

$$
\begin{aligned}
\Phi= & \left(\psi_{30}-\psi_{3 L}\right) L\left(-3 \psi_{2 L}^{\prime}+\psi_{2 L}^{\prime \prime} L\right) \\
& +\psi_{2 L}\left(-6 \psi_{4 L}^{\prime}+L\left(-3 \psi_{3 L}^{\prime}+3 \psi_{4 L}^{\prime \prime}+\psi_{3 L}^{\prime \prime} L-\psi_{4 L}^{\prime \prime \prime} L\right)\right) \\
& +\psi_{20}\left(6 \psi_{4 L}^{\prime}+L\left(3 \psi_{3 L}^{\prime}-3 \psi_{4 L}^{\prime \prime}-\psi_{3 L}^{\prime \prime} L+\psi_{L L}^{\prime \prime \prime}\right)\right) \\
\psi_{20}= & \left.\psi_{2}\right|_{x=0}, \psi_{2 L}=\left.\psi_{2}\right|_{x=L}, \psi_{20}^{\prime}=\left.\psi_{2}^{\prime}\right|_{x=0}, \psi_{2 L}^{\prime}=\left.\psi_{2}^{\prime}\right|_{x=L}, \psi_{20}^{\prime \prime}=\left.\psi_{2}^{\prime \prime}\right|_{x=0}, \psi_{2 L}^{\prime \prime}=\left.\psi_{2}^{\prime \prime}\right|_{x=L} \\
\psi_{30}= & \left.\psi_{3}\right|_{x=0}, \psi_{3 L}=\left.\psi_{3}\right|_{x=L}, \psi_{30}^{\prime}=\left.\psi_{3}^{\prime}\right|_{x=0}, \psi_{3 L}^{\prime}=\left.\psi_{3}^{\prime}\right|_{x=L}, \psi_{30}^{\prime \prime}=\left.\psi_{3}^{\prime \prime}\right|_{x=0}, \psi_{3 L}^{\prime \prime}=\left.\psi_{3}^{\prime \prime}\right|_{x=L} \\
\psi_{40}= & \left.\psi_{4}\right|_{x=0}, \psi_{4 L}=\left.\psi_{4}\right|_{x=L}, \psi_{40}^{\prime}=\left.\psi_{4}^{\prime}\right|_{x=0}, \psi_{4 L}^{\prime}=\left.\psi_{4}^{\prime}\right|_{x=L}, \psi_{40}^{\prime \prime}=\left.\psi_{4}^{\prime \prime}\right|_{x=0}, \psi_{4 L}^{\prime \prime}=\left.\psi_{4}^{\prime \prime}\right|_{x=L} \\
\psi_{40}^{\prime \prime \prime}= & \left.\psi_{4}^{\prime \prime \prime}\right|_{x=0}, \psi_{4 L}^{\prime \prime \prime}=\left.\psi_{4}^{\prime \prime \prime}\right|_{x=L}
\end{aligned}
$$

\section{Governing Equations}

The governing equation can be derived via Hamilton's principle as follow:

$$
\delta H=\int_{t_{1}}^{t_{2}}\left(\delta S_{E}-\delta K_{E}-\delta W_{E}\right) d t=0
$$

where, $\delta H, \delta S_{E}, \delta K_{E}$ and $\delta W_{E}$ are the variation of total energy, strain energy, kinetic energy and external work of the beam, respectively.

The undamped equilibrium equation can be obtained by substituting (5) and (13) into the variational equations of (18), which results in

$$
\mathbf{K d}-\mathbf{M} \ddot{\mathbf{d}}-\mathbf{f}=\mathbf{0}
$$

where, $\mathbf{K}, \mathbf{M}, \mathbf{f}, \mathbf{d}$ and $\ddot{\mathbf{d}}$ are the stiffness matrix, mass matrix, loading vector, general displacement vector and general acceleration vector, respectively. The stiffness matrix, mass matrix and loading vector are given by

$$
\begin{aligned}
& \mathbf{K}=\int_{0}^{L}\left\{\begin{array}{c}
\mathbf{N}_{u}^{\prime} \\
\left(\mathbf{N}_{v}^{\prime}-\mathbf{N}_{\theta}\right) \\
\mathbf{N}_{\theta}^{\prime}
\end{array}\right\}^{T}\left[\begin{array}{ccc}
E(x) A(x) & 0 & 0 \\
0 & \kappa G(x) A(x) & 0 \\
0 & 0 & E(x) I(x)
\end{array}\right]\left\{\begin{array}{c}
\mathbf{N}_{u}^{\prime} \\
\left(\mathbf{N}_{v}^{\prime}-\mathbf{N}_{\theta}\right) \\
\mathbf{N}_{\theta}^{\prime}
\end{array}\right\} d x \\
& \mathbf{M}=\int_{0}^{L}\left\{\begin{array}{l}
\mathbf{N}_{u} \\
\mathbf{N}_{v} \\
\mathbf{N}_{\theta}
\end{array}\right\}^{T}\left[\begin{array}{ccc}
\rho(x) A(x) & 0 & 0 \\
0 & \rho(x) A(x) & 0 \\
0 & 0 & \rho(x) I(x)
\end{array}\right]\left\{\begin{array}{l}
\mathbf{N}_{u} \\
\mathbf{N}_{v} \\
\mathbf{N}_{\theta}
\end{array}\right\} d x \\
& \mathbf{f}=\left\{\begin{array}{cc}
\mathbf{N}_{u 1} & P_{1} \\
\mathbf{N}_{v 1} & Q_{1} \\
\mathbf{N}_{\theta 1} & M_{1} \\
\mathbf{N}_{u 2} & P_{2} \\
\mathbf{N}_{v 2} & Q_{2} \\
\mathbf{N}_{\theta 2} & M_{2}
\end{array}\right\}+\int_{0}^{L}\left\{\begin{array}{cc}
\mathbf{N}_{u 1} & p(x) \\
\mathbf{N}_{v 1} & q(x) \\
\mathbf{N}_{\theta 1} & m(x) \\
\mathbf{N}_{u 2} & p(x) \\
\mathbf{N}_{v 2} & q(x) \\
\mathbf{N}_{\theta 2} & m(x)
\end{array}\right\} d x
\end{aligned}
$$


where, $P_{i}, Q_{i}$ and $M_{i}$ are the concentrated axial load, transversal load and moment at node $i$, respectively. The terms $p(x), q(x)$ and $m(x)$ are the distributed axial load, transversal load and moment, respectively.

In constructing the matrices and vector of (20-22), the Gaussian quadrature integration scheme is recommended. Also, the shape functions $\psi_{1}, \psi_{2}, \psi_{3}, \psi_{4}$ and their derivatives inside the equations can be evaluated numerically by using the Gaussian quadrature integration scheme. These shape functions will carry on the non-uniformity and inhomogeneity characteristics of the beam.

\section{Numerical Examples}

In this section, illustrative examples are solved by using the exact displacement functions derived in previous sections to show the validity and novelty of the present study. Comparisons with the numerical results reported by other studies are highlighted.

\subsection{Taper Cantilever Beam}

A homogeneous non-dimensional unit tapered cantilever beam loaded at the free end tip as shown in Fig. 1 is considered.

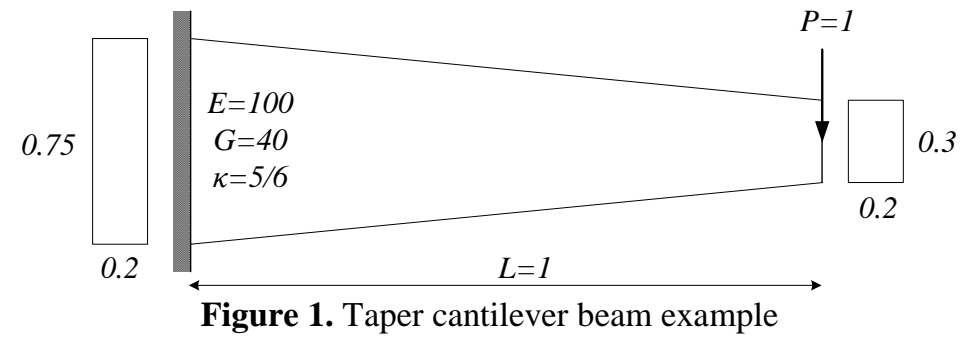

The beam is analyzed by using one element by using the exact displacement functions to construct the stiffness matrices of the static equilibrium equation.

Table 1. Results of static analysis of taper cantilever beam example

\begin{tabular}{|c|c|c|c|c|}
\hline \multirow{2}{*}{$\begin{array}{c}\text { Shear Deformation } \\
\text { Consideration }\end{array}$} & \multicolumn{2}{|c|}{ Reaction at fixed end } & \multicolumn{2}{c|}{ Vertical displacement at free end } \\
\cline { 2 - 5 } & Shearing Force & Bending Moment & Present Study & {$[25]$} \\
\cline { 2 - 5 } & $Q$ & $M$ & & \multicolumn{2}{|c|}{$w$} \\
\hline Considered & 1.0 & 1.0 & 1.203 & 1.203 \\
\hline Not considered & 1.0 & 1.0 & 0.8977 & 0.897 \\
\hline
\end{tabular}

As shown in Table 1., the reaction forces and displacement resulting from the shear deformation consideration are in good agreement with the theoretical results of [25].

\subsection{Various Taper Clamped Beam}

A homogeneous non-dimensional unit various tapered clamped beam loaded at the mid-span as shown in Fig. 2 is considered. Two different heights $h$ and various values of the lengths $L$ are analyzed.

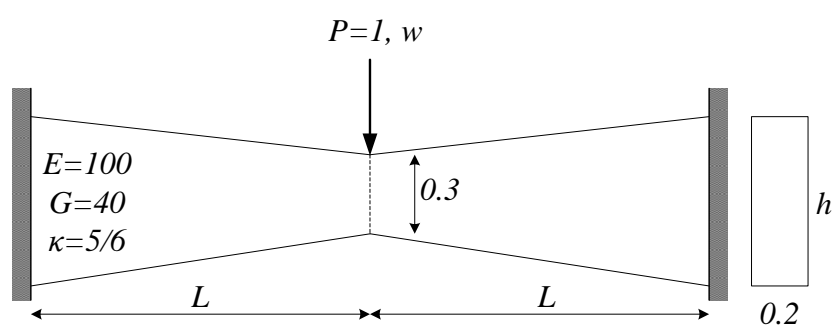

Figure 2. Clamped taper beam example

Deflections which include shear deformation $\left(w_{s}\right)$ and neglect shear deformation $\left(w_{n s}\right)$ at the mid-span of the beams are listed in Tables 2, respectively. The present computed deflections are compared with the results of [28].

Only using two element divisions, the present results gave an excellent agreement with the reference (twelve elements) which can be observed from the table. 
Table 2. Deflection of clamped taper beam example

\begin{tabular}{|c|c|c|c|c|c|c|c|c|}
\hline & \multicolumn{4}{|c|}{$h=0.6$} & \multicolumn{4}{c|}{$h=0.9$} \\
\hline \multirow{2}{*}{$L$} & \multicolumn{2}{|c|}{$w_{s}$} & \multicolumn{2}{c|}{$w_{n s}$} & \multicolumn{2}{c|}{$w_{s}$} & \multicolumn{2}{c|}{$w_{n s}$} \\
\cline { 2 - 10 } & Present & {$[28]$} & Present & {$[28]$} & Present & {$[28]$} & Present & {$[28]$} \\
\hline 1 & 0.4670 & 0.468 & 0.2943 & 0.294 & 0.2748 & 0.274 & 0.1368 & 0.137 \\
\hline 2 & 2.6991 & 2.700 & 2.3541 & 2.354 & 1.3708 & 1.370 & 1.0950 & 1.096 \\
\hline 3 & 8.4647 & 8.464 & 7.9376 & 7.944 & 4.1105 & 4.110 & 3.6991 & 3.698 \\
\hline 4 & 19.525 & 19.524 & 18.8324 & 18.831 & 9.315 & 9.315 & 8.7668 & 8.766 \\
\hline 5 & 37.6454 & 37.645 & 36.7784 & 36.779 & 17.8088 & 17.807 & 17.1203 & 17.120 \\
\hline
\end{tabular}

\subsection{Free Vibration of Various Boundary Conditions of Taper Beams}

Various boundary conditions of taper beams as shown in Fig. 3. The geometry and material data of the beam are given in the figure.

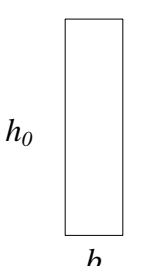

b
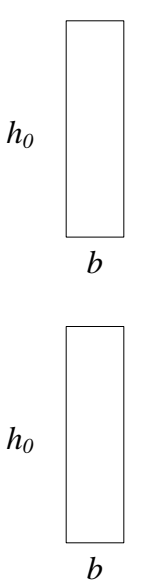

b
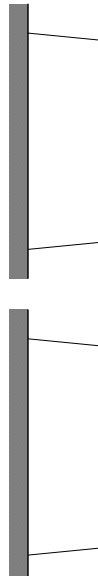

a) Clamped-Free $(C-F)$

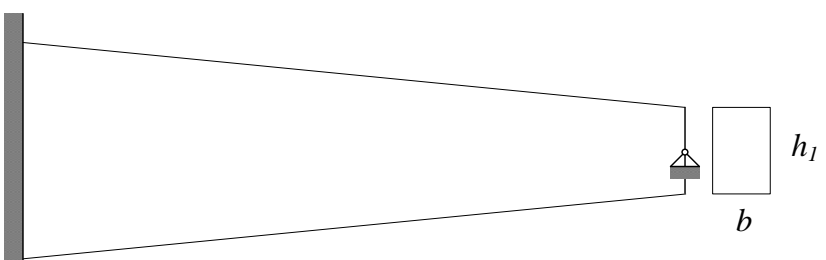

c) Clamped-Pinned (C-P)
$E=210 \mathrm{Gpa}$

$E /(\kappa G)=3.12$

$K=2 / 3$

$L=0.8660254 m$

$\rho=7850 \mathrm{~kg} / \mathrm{m}^{3}$

$r^{2}=I_{0}\left(A_{0} L^{2}\right)=0.08$

Figure 3. Various boundary conditions of taper beam free vibration example

The cross-session is rectangular with constant width $b$ and linear-varying height $h$ defined as

$$
\beta=\frac{h_{1}-h_{0}}{h_{0}}
$$

The non-dimensional frequencies of the beam that are defined as

$$
\mu_{i}=\omega_{i} \sqrt{\frac{\rho A_{0} L^{4}}{E I_{0}}}
$$

From Table 3, the results of the non-dimensional frequencies of the present formulation show lower convergence results under various boundary conditions compared to the results in [15]. In the free vibration problem, the beam element should be divided into sub-elements to accommodate the free degree of freedoms. In this study, only four element divisions for the beam were employed to obtain the good results. 
Table 3. Non-dimensional natural frequency of tapered beam with various boundary conditions

\begin{tabular}{|c|c|c|c|c|c|c|c|}
\hline$\beta$ & $\begin{array}{c}\text { Boundary } \\
\text { Condition }\end{array}$ & & $\mu_{1}$ & $\mu_{2}$ & $\mu_{3}$ & $\mu_{4}$ & $\mu_{5}$ \\
\hline 0.0 & \multirow{2}{*}{ C-F } & Present & 3.22720 & 14.47780 & 31.50240 & 48.19680 & 62.87280 \\
\cline { 3 - 8 } & {$[15]$} & 3.22713 & 14.46893 & 31.50254 & 47.90902 & 62.34702 \\
\cline { 3 - 8 } & \multirow{2}{*}{ C-P } & Present & 11.08680 & 27.17590 & 45.11830 & 59.44520 & 63.59720 \\
\cline { 3 - 8 } & {$[15]$} & 11.08250 & 27.11438 & 44.84353 & 59.20303 & 63.33950 \\
\cline { 3 - 8 } & \multirow{2}{*}{ C-C } & Present & 13.83890 & 28.58680 & 45.95580 & 61.42410 & 68.86960 \\
\cline { 3 - 8 } & \multirow{2}{*}{ C-F } & Present & 3.34530 & 14.32890 & 30.91900 & 48.13030 & 65.05240 \\
\cline { 3 - 8 } & & {$[15]$} & 3.33065 & 14.28921 & 30.71080 & 47.75021 & 64.99695 \\
\cline { 3 - 8 } & \multirow{2}{*}{ C-P } & Present & 10.75020 & 26.31190 & 44.03880 & 62.05360 & 68.56940 \\
\cline { 3 - 8 } & & {$[15]$} & 10.68689 & 26.10717 & 43.59072 & 61.65596 & 68.42075 \\
\cline { 3 - 8 } & \multirow{2}{*}{ C-C } & Present & 13.31860 & 27.95770 & 45.12020 & 61.90550 & 72.36990 \\
\cline { 3 - 8 } & & {$[15]$} & 13.22227 & 27.77822 & 44.69713 & 61.80658 & 72.55473 \\
\hline
\end{tabular}

\subsection{Functionally graded beam}

The vibration of a uniform cross-section of an inhomogeneous Functionally Graded Material (FGM) beam shown in Fig. 4 is investigated. The beam is made of two materials from the left to the right ends with a constant value of mass density, the effective elastic modulus, $E$ and shear modulus, $G$ of the beam are assumed to vary in the beam axis direction according to the following

$$
\begin{aligned}
& E(x)=\left(E_{\text {left }}-E_{\text {right }}\right)\left(1-\frac{x}{L}\right)+E_{\text {right }} \\
& G(x)=\left(G_{\text {left }}-G_{\text {right }}\right)\left(1-\frac{x}{L}\right)+G_{r i g h t}
\end{aligned}
$$

The Simply-Supported (S-S) rectangular - cross-section beam with geometric data (width $b=0.4 \mathrm{~m}$; height $h=0.9 \mathrm{~m}$ and the total length $L=20 \mathrm{~m}$ ) is employed in the computation of fundamental frequencies.

The results (with only one element division) are compared with [12] which is computed with ten element divisions. Very good agreement between the frequencies obtained in the present work with that of the reference is given in Table 4 where $E_{r}=E_{\text {lefi }} / E_{\text {right }} ; \mu_{1}, \mu_{2}$ are respectively the first and the second nondimensionalized fundamental frequencies of the beam. The non-dimensionalized fundamental frequency is given by

$$
\mu_{i}=\omega_{i} \sqrt{\frac{\rho A L^{4}}{E_{l e f t} I}}
$$

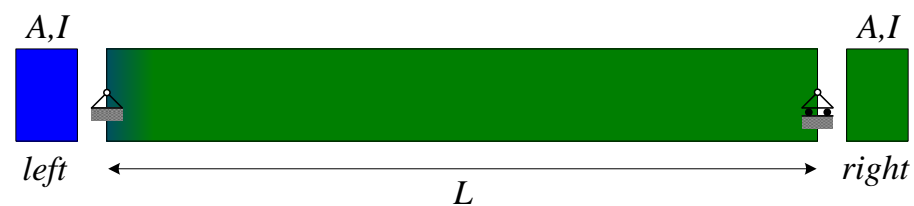

Figure 4. Simply supported rectangular FGM beam example

Table 4. Non-dimensional fundamental frequencies of FGM beam example

\begin{tabular}{|c|c|c|c|c|}
\hline \multirow{2}{*}{$E_{R}$} & \multicolumn{2}{|c|}{$\mu_{1}$} & \multicolumn{2}{c|}{$\mu_{2}$} \\
\cline { 2 - 5 } & Present & {$[12]$} & Present & {$[12]$} \\
\hline 0.25 & 2.7493 & 2.74822 & 5.4402 & 5.43722 \\
\hline 0.5 & 2.9092 & 2.9056 & 5.7731 & 5.7685 \\
\hline 1 & 3.1378 & 3.1350 & 6.2353 & 6.2317 \\
\hline 2 & 3.4597 & 3.4554 & 6.8626 & 6.8599 \\
\hline 4 & 3.8900 & 3.8866 & 7.6903 & 7.6893 \\
\hline
\end{tabular}




\section{Conclusions}

Exact shape functions of a non-uniform and inhomogeneous Timoshenko beam element has been formulated. These shape functions can be implemented in finite-element codes to create the mass and stiffness matrices within the context of Timoshenko beam element where the shear deformation can be taken into account in the analyses. The correctness of the formulated shape functions was verified through the numerical examples of the static and free vibration analyses of non-uniform and inhomogeneous (FGM) beam elements. With the formulated shape functions, highly accurate results can be obtained by using the least element number of element division.

\section{References}

[1] D.K. Nguyen, B.S. Gan and T.H. Le, Dynamic Response of Non-Uniform Functionally Graded Beams Subjected to a Variable Speed Moving Load, Journal of Computational Science and Technology, 7(1), 2013, 12-27.

[2] D.K. Nguyen and B.S. Gan, Large deflections of tapered functionally graded beams subjected to end forces, Applied Mathematical Modelling, 38(11-12), 2013, 3054-3066.

[3] D.K. Nguyen, B.S. Gan and T.H. Trinh, Geometrically nonlinear analysis of planar beam and frame structures made of functionally graded material, Structural Engineering and Mechanics, 49(6), 2014, 727-743.

[4] T.H. Le, B.S. Gan, T.H. Trinh and D.K. Nguyen, Finite element analysis of multi-span functionally graded beams under a moving harmonic load, Mechanical Engineering Journal, 1(3), 2014, 1-13.

[5] B.S. Gan and D.K. Nguyen, Large Deflection Analysis of Functionally Graded Beams Resting on a Two-Parameter Elastic Foundation, Journal of Asian Architecture and Building Engineering, 13(3), 2014, 649-656.

[6] D.K. Nguyen, B.S. Gan, T.H. Trinh, and S. Alexandrov, Post-buckling Behavior of Elastic-Plastic Functionally Graded Beams Subjected to Eccentric Axial Load, Journal of Multidisciplinary Engineering Science and Technology, 2(5), 2015, 1129-1135.

[7] A.L. Han, B.S. Gan, S. As'ad and M.M.A. Pratama, Parametric Study of the Load Carrying Capacity of Functionally Graded Concrete of Flexural Members, International Journal of Engineering and Technology Innovation, 5(4), 2015, $233-241$

[8] T.H. Trinh, D.K. Nguyen, B.S. Gan and S. Alexandrov, Post-buckling responses of elastoplastic FGM beams of nonlinear elastic foundation, Structural Engineering and Mechanics, 58(3), 2016, 515-532.

[9] D.K. Nguyen, T.T. Tran, B.S. Gan and V.T. Bui, Influences of Dynamic Moving Forces on the Functionally Graded PorousNonuniform Beams, International Journal of Engineering and Technology Innovation, 6(3), 2016, 173-189.

[10] T.H. Trinh, V.T. Bui, N.H. Nguyen, D.K. Nguyen and B.S. Gan, Dynamic Behavior of Functionally Graded Beams in Thermal Environment due to a Moving Harmonic Load, International Journal of Mechanical Systems Engineering, 2(119), $2016,1-8$.

[11] B.S. Gan, D.K. Nguyen and T.H. Le, Effect of Intermediate Elastic Support on Vibration of Functionally Graded Euler-Bernoulli Beams Excited by a Moving Point Load, Journal of Asian Architecture and Building Engineering, 16(2), 2017, 363-369.

[12] B.S. Gan, T.H. Trinh, T.H. Le and D.K. Nguyen, Dynamic response of non-uniform Timoshenko beams made of axially FGM subjected to multiple moving point loads, Structural Engineering and Mechanics, 53(5), 2015, 981-995.

[13] A.Y.T. Leung, W.E. Zhou, C.W. Lim, R.K.K. Yuen and U. Lee, Dynamic stiffness for piecewise non-uniform Timoshenko column by power series - part I: Conservative axial force, International Journal for Numerical Methods in Engineering, 51(5), 2001, 505529.

[14] A.Y.T. Leung, W.E. Zhou, C.W. Lim, R.K.K. Yuen and U. Lee, Dynamic stiffness for piecewise non-uniform Timoshenko column by power series - part II: Follower force, International Journal for Numerical Methods in Engineering, 51(5), 2001, 531-552.

[15] R. Attarnejad, S.J. Semnani and A. Shahba, Basic displacement functions for free vibration analysis of non-prismatic Timoshenko beams, Finite Elements in Analysis and Design, 46(10), 2010, 916-929.

[16] T.H. Trinh, T. Hibino and B.S. Gan, Consistent Shape Function for Free Vibration of Linearly Tapered Timoshenko Beam Element, Bulletin of Nihon University College of Engineering, 57(1), 2015, 7-14.

[17] T.H. Trinh and B.S. Gan, Development of Consistent Shape Functions for linearly solid tapered Timoshenko Beam, Journal of Structural and Construction Engineering, 80(713), 2015, 1103-1111.

[18] S.P. Timoshenko, On the correction for shear of the differential equation for transverse vibrations of prismatic bars, Philosophical Magazine, 41, 1921, 744-746.

[19] S.P. Timoshenko, On the transverse vibrations of bars of uniform cross-section, Philosophical Magazine, 43, 1922, $125-131$.

[20] J.S. Archer, Consistent mass matrix formulation for structural analysis using finite element techniques, American Institute of Aeronautics and Astronautics Journal, 3, 1965, 1910-1918.

[21] R.T. Severn, Inclusion of shear deflection in the stiffness matrix for a beam element, The Journal of Strain Analysis for Engineering Design, 5(4), 1970, 239-241.

[22] D.M. Egle, An approximate theory for transverse shear deformation and rotary inertia effects in vibrating beams, NASA CR-1317, 1969.

[23] Z. Friedman and J.B. Kosmatka, An Improved Two-Node Timoshenko Beam Finite Element, Computers \& Structures, 47(3), 1993, 473-481.

[24] J.B. Kosmatka, An improved two-node finite element for stability and natural frequencies of axial-loaded Timoshenko beams, Computers \& Structures, 57(1), 1995, 141-149.

[25] M. Eisenberger, Explicit stiffness matrices for non-prismatic members, Computers \& Structures, 20(4), 1985, 715-720

[26] B.S. Gan, T.H. Trinh and D.K. Nguyen, Post-buckling behaviour of axially FGM planar beams and frames, Procedia Engineering, $171,2017,147-158$

[27] J.N. Reddy, Energy principles and variational methods in applied mechanics (Second edition, Hoboken, NJ: John Wiley \& Sons, 2002).

[28] M. Eisenberger, Stiffness matrices for non-prismatic members including transverse shear, Computers \& Structures, 40(4), 1991, 831-835. 